Chang-Seok Ki · Jong Seo Hong • Gyu-Young Jeong Kyoung Ju Ahn • Kwang-Mo Choi • Duk-Kyung Kim Jong-Won Kim

\title{
Identification of lamin A/C (LMNA) gene mutations in Korean patients with autosomal dominant Emery-Dreifuss muscular dystrophy and limb-girdle muscular dystrophy 1B
}

Received: November 19, 2001 / Accepted: February 8, 2002

\begin{abstract}
Mutations in the LMNA gene encoding lamins A and $\mathrm{C}$ by alternative splicing have been found to cause at least four different kinds of genetic disorders: autosomal dominant Emery-Dreifuss muscular dystrophy (EDMD2; MIM 181350); limb-girdle muscular dystrophy type 1B (LGMD1B; MIM 159001); dilated cardiomyopathy type 1A (CMD1A; MIM 115200); and familial partial lipodystrophy (FPLD; MIM 151660). Recently, we have studied two Korean patients with atrioventricular conduction defects. They had variable extents of muscular dystrophy; one patient was diagnosed with EDMD2 and the other with LGMD1B. We performed a mutation analysis of the $L M N A$ gene by direct sequencing and found two different missense mutations: R249Q and R377L, in the EDMD2 and LGMD1B patient, respectively. The R249Q mutation is located within the central rod domain of the $L M N A$ gene, and has been described in at least five unrelated sporadic EDMD2 patients. On the other hand, the R377L mutation, also located within the rod domain, is a novel mutation, although a histidine substitution instead of leucine $(\mathrm{R} 377 \mathrm{H})$ has been reported previously in an LGMD1B patient. To
\end{abstract}

C.-S. Ki · K.-M. Choi $\cdot$ J.-W. Kim ${ }^{1}(\square)$

Department of Clinical Pathology, Sungkyunkwan University School of Medicine, Samsung Medical Center, 50 Ilwon-dong,

Kanagnam-ku, Seoul, 135-710, Korea

Tel. +82-2-3410-2705; Fax +82-2-3410-2719

e-mail: culture@med.skku.ac.kr

J.S. Hong D.-K. Kim ${ }^{1}$

Department of Internal Medicine, Sungkyunkwan University School of Medicine, Seoul, Korea

G.-Y. Jeong

Department of Clinical Pathology, Seoul Clinical Laboratories, Seoul Medical Science Institute, Seoul, Korea

K.J. Ahn

Department of Internal Medicine, Han-il General Hospital, Seoul, Korea

${ }^{1}$ These two authors contributed equally to this work. our knowledge, this is the first report of $L M N A$ gene mutations in Korean patients with EDMD2 and LGMD1B.

Key words Lamin $\mathrm{A} / \mathrm{C} \cdot L M N A \cdot$ Autosomal dominant Emery-Dreifuss muscular dystrophy - EDMD2 Limb-girdle muscular dystrophy 1B $\cdot$ LGMD1B

\section{Introduction}

Lamins are members of the intermediate filament protein family, and they are major components of the nuclear envelope. Like other intermediate filament proteins, nuclear lamins have a central $\alpha$-helical coiled-coil domain, flanked by globular amino-terminal head and carboxyl-terminal tail domains. Lamin $\mathrm{A}$ and $\mathrm{C}$ are splicing variants encoded by the lamin A/C (LMNA; MIM 150330) gene and share the first 566 amino acids (Stuurman et al. 1998; Nagano and Arahata 2000). In 1999, Bonne et al. (1999) found that a mutation in the LMNA gene is the cause of an autosomal dominant Emery-Dreifuss muscular dystrophy (EDMD2; MIM 181350). Soon after that discovery, other disorders such as dilated cardiomyopathy type 1A (CMD1A; MIM 115200) (Fatkin et al. 1999), limb-girdle muscular dystrophy type 1B (LGMD1B; MIM 159001) (Muchir et al. 2000), and familial partial lipodystrophy (FPLD; MIM 151660) (Shackleton et al. 2000) were found to be allelic disorders of the same gene.

Among these disorders related to $L M N A$ gene mutations, EDMD2 is characterized by early contractures of elbows and Achilles tendons, slowly progressive muscle wasting and weakness, and cardiomyopathy with a variable degree of conduction-system disease, which are identical symptoms to those of classic X-linked EDMD (MIM 310300) (Emery 2000). On the other hand, LGMD1B is slowly progressive, with age-related atrioventricular (AV) cardiac conduction disturbance, dilated cardiomyopathy, and the absence of early contractures (van der Kooi et al. 1996).

Recently, we have studied two Korean patients with atrioventricular conduction defects. Although they had 
similar cardiac problems, the extent of muscular dystrophy showed such wide variation that one patient was diagnosed with EDMD2 and the other with LGMD1B. To test whether these patients had $L M N A$ mutations or not, we performed a mutation analysis of the LMNA gene and found two different missense mutations. Here, we describe the results of the mutation analysis as well as clinical and laboratory findings relating to the patients.

\section{Subjects and methods}

\section{Subjects}

Two Korean patients and their family members were examined for mutations in the $L M N A$ gene. The diagnoses of the patients were made on the basis of clinical symptoms, family histories, physical as well as neurological examinations, cardiological investigations including 12-lead electrocardiography (ECG), 24-hour ECG monitoring and echocardiography, electrophysiologic studies including nerve conduction velocity (NCV) and electromyography (EMG), laboratory studies of plasma creatine kinase (CK) enzyme activity, and muscle biopsy. Information on deceased family members as well as on other family members who were suspected to be affected was obtained from probands.

\section{Case reports}

The patient of case 1 was a 28 -year-old woman presented to the cardiology department complaining of intermittent dizziness and palpitation. She developed progressive wasting of the lower leg muscles at age 3 years and began to have difficulty walking at age 6 years. A physical examination demonstrated severe wasting of the bilateral shoulder girdle, proximal arm, and proximal and distal leg muscles. A marked limitation of cervical flexion, both elbow and Achilles tendon contractures, and equinus deformities of both feet were also noted.

The plasma CK level was 254 IU/1 (reference interval: 22-268 IU/l), and was all of the skeletal-muscle (CK-MM) type. NCV was not impaired but an EMG revealed an early recruitment pattern, which was consistent with chronic myogenic disorder. The ECG showed sinus pause and a first degree AV block. A Morbitz type I AV block and a high-egree AV block-associated atrial tachycardia (120 beats/min) were noted in a 24-hour ECG monitoring study. Echocardiography revealed normal systolic function and cavity size of the left ventricle, but a restrictive pattern was noted.

An elder sister (Fig. 1A) with similar clinical features to the patient died of an unknown cause at age 28 years. No other family member had any clinical symptoms or signs of the disease. The patient was diagnosed as having EDMD2, and a VVI-type permanent pacemaker was implanted to prevent sudden death associated with high-degree AV block.

The patient of case 2 was a 41-year-old woman who had suffered from ankle contractures for 25 years. She had gait disturbance and difficulty climbing stairs due to ankle contractures as well as proximal leg weakness. At the age of 39 , she experienced a loss of consciousness for $30 \mathrm{~min}$, which resulted in paraplegia for one year. When she visited our hospital to relieve the ankle contractures, a neurological evaluation revealed limb-girdle muscle wasting, particularly proximal arms and thighs.

The serum CK level was 55 IU/l (reference interval: 26140 IU/l). NCV studies were normal, but EMG revealed short-duration and small-amplitude polyphasic motor unit potentials over the biceps, vastus medialis, orbicularis oris, and gluteus maximus muscles. Spontaneous activities such as fibrillation potentials were absent. A muscle biopsy demonstrated variation in fiber size with round atrophic and some hypertrophic fibers and an increased amount of internal nuclei. Atrial fibrillation was noticed on ECG, and the longest R-R interval was $2060 \mathrm{msec}$ in a 24-hour ambulatory ECG. Echocardiography showed dilated cardiomyopathy (left ventricle ejection fraction: $40 \%$ ).

Two elder sisters had died at age 20 and 38 years due to heart problems, and another elder sister had similar problems as the patient (Fig. 1B). This patient was diagnosed as having LGMD1B, and a VVI-type pacemaker was also implanted.

\section{Mutation analysis}

The patient with EDMD2 and her mother, two elder sisters, and brother were tested for the LMNA mutation. Because
Fig. 1A,B. Pedigrees of families with EDMD2 (A) and LGMD1B (B). Squares indicate males, circles indicate females, arrows indicate probands, and slash marks indicate deceased family members. Open symbols indicate unaffected family members, darkened symbols indicate affected family members, and shaded symbols indicate family members who are or were probably affected

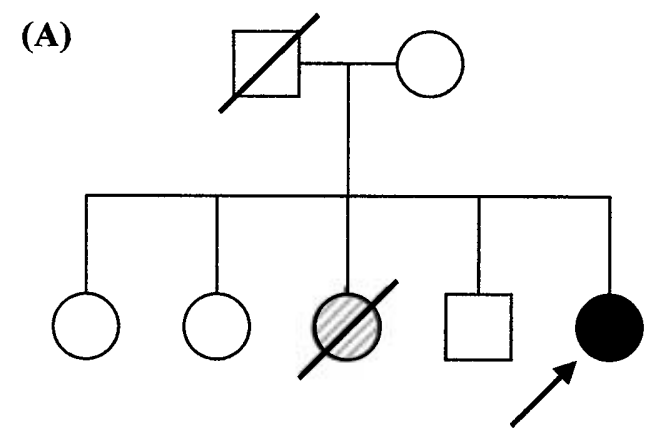

(B)

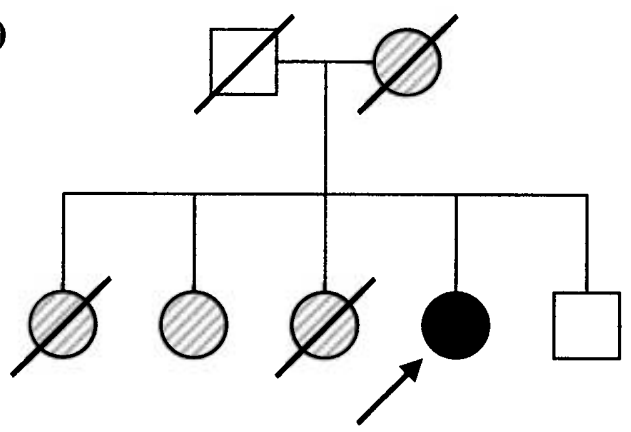


all family members of the patient with LGMD1B refused mutation analysis, only the patient was included in the study. After we had ostained informed consent, genomic DNA was isolated from peripheral blood leukocytes using a Wizard genomic DNA purification kit following the manufacturer's instruction (Promega, Madison, WI, USA). All 12 exons of the LMNA gene were amplified by polymerase chain reaction (PCR) by using specific primers provided by Seidman CE (http://genetics.med.harvard.edu/ seidman/lamin.html), as described previously (Fatkin et al. 1999). PCR amplicons were extracted from an agarose gel using a gel extraction kit (Qiagen, Valencia, CA, USA) and then subjected to direct sequencing analysis on an automated ABI Prism 3100 Genetic Analyzer (Applied Biosystems, Foster City, CA, USA) with a BigDye Terminator Cycle Sequencing Ready Reaction kit, version 2.0. (Applied Biosystems). The presence of each nucleotide alteration detected by direct sequencing was confirmed by restriction endonuclease digestion if possible.

\section{Results and discussion}

In the patient with EDMD2, a $\mathrm{G}$ to $\mathrm{A}$ transition in exon 4 of the $L M N A$ gene (c.746G $>\mathrm{A}$; R249Q) was observed (Fig. 2), which was not found in any of the tested family members. In the patient with LGMD1B, a $\mathrm{G}$ to $\mathrm{T}$ transversion in exon 6 of the LMNA gene (c.1130G > T; R377L) was found (Fig. 2). Both mutations were confirmed by restriction endonuclease digestion with AciI. The R377L mutation was not found in 110 unrelated control subjects. Since the R249Q mutation found in the EDMD2 patient was not novel, we did not test the mutation in control subjects.

The R249Q mutation has been reported previously in at least five EDMD2 patients (Bonne et al. 2000; Di Barletta et al. 2000; Brown et al. 2001). Different from our patient, four of them were male (one unknown) and three of them were diagnosed as having X-linked EDMD (Di Barletta et al. 2000). All EDMD2 patients with the R249Q mutation of the $L M N A$ gene had heart problems. Although we could
(A)

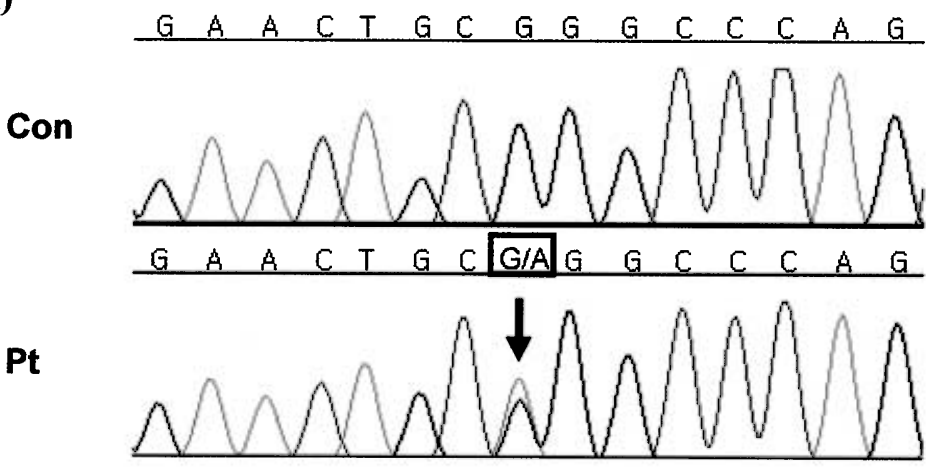

(C)

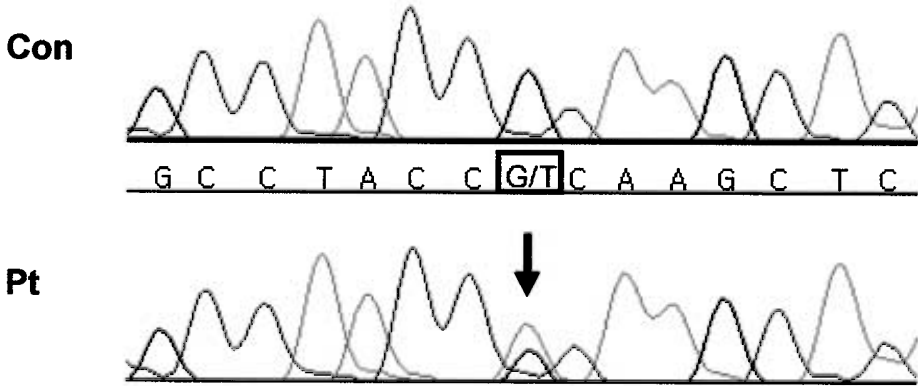

(B)

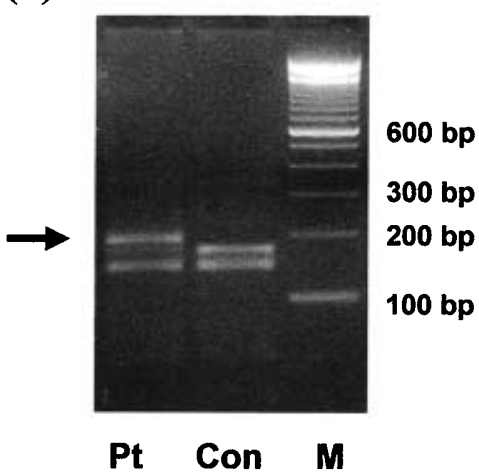

(D)

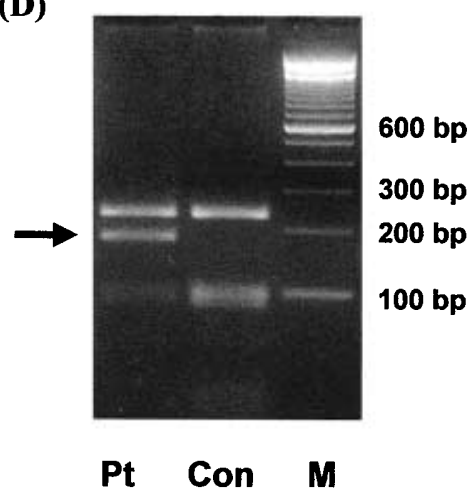

Fig. 2A-D. Identification of $L M N A$ mutations in patients with EDMD2 (A and B) and LGMD1B (C and D). A Direct sequencing demonstrates a heterozygous $\mathrm{G}$ to $\mathrm{A}$ transition (arrow; c.746G $>\mathrm{A}$ ) within exon 4, resulting in a R249Q missense mutation in the EDMD2 patient. B Polymerase chain reaction-restriction fragment length polymorphism (PCR-RFLP) analysis confirms the R249Q mutation, which destroys an AciI site creating a 180-bp band (arrow). C A heterozygous $\mathrm{G}$ to $\mathrm{T}$ transversion within exon 6 (arrow; c.1130G $>\mathrm{T}$ ) resulting in an R377L missense mutation was observed in the LGMD1B patient. D PCR RFLP analysis also confirms the R377L mutation, which destroys an AciI site creating a 179-bp band (arrow). Con, wild-type control; Pt, EDMD2 or LGMD1B patient; M, 100-bp size marker 
not perform mutation analysis of the patient's father, no family member other than the deceased sister showed clinical presentations of EDMD2. However, it is not clear whether the patient's sister died from complications of EDMD2 or not.

Based on the patient's family history, either the mutation arose de novo in the patient's generation, or germline mosaicism may explain the occurrence of the R249Q mutation in the patient. The possibility that it is a recessive trait was excluded because the R249Q mutation has been found in at least five EDMD2 patients and because there was no genetic variation other than the heterozygous R249Q mutation in our patient or in tested family members.

Up to now, four different $L M N A$ gene mutations were reported to be associated with LGMD1B. Therefore, this is the fifth report of an LGMD1B patient carrying an $L M N A$ gene mutation. Known $L M N A$ mutations causing LGMD1B are an in-frame deletion in exon 3 (c.334336delGAG; delK208) (Muchir et al. 2000), a missense mutation in exon 6 (R377H and R377L) (Muchir et al. 2000), a missense mutation in exon 8 (Y481H) (Kitaguchi et al. 2001), and a splice donor site mutation in intron 9 (IVS9+5G $>$ C) (Muchir et al. 2000).

The delK208 mutation is localized in the central rod domain that is implicated in the dimerization of lamins (Heald and McKeon 1990). The R377H and R377L mutations are located among the 32 amino acids highly conserved in all intermediate filaments at the end of the $\alpha$-helix rod domain (Muchir et al. 2000; Genschel and Schmidt 2000). By a systematic mutagenesis of this 32-amino-acid region, Heald and McKeon (1990) showed that both the R377H and R377P mutations abolish the assembly of normal nuclear lamina. The $\mathrm{Y} 481 \mathrm{H}$ mutation changes an amino acid in the carboxyl-terminal tail domain of lamin $\mathrm{A} / \mathrm{C}$, which has been conserved through different species and different lamins (Kitaguchi et al. 2001). The IVS9+5G $>C$ mutation also affects the carboxyl-terminal tail domain of lamin $\mathrm{A} / \mathrm{C}$, which leads to an aberrant $L M N A$ transcript with the retention of intron 9 and a frameshift at position 536 (Muchir et al. 2000). It is noteworthy that two out of five mutations causing LGMD1B were found at the same codon (R377). Therefore, R377 may be an important codon strictly related to the LGMD1B phenotype.

Different from EDMD2, all LGMD1B patients with $L M N A$ gene mutations reported so far were familial cases. They showed similar clinical features with slowly progressive limb-girdle weakness, the absence of early contractures, age-related atrioventricular cardiac conduction disturbance, and dilated cardiomyopathy (van der Kooi et al. 1996; Muchir et al. 2000; Kitaguchi et al. 2001). Our patient in the present study was also a familial case and showed very similar clinical features as described previously.

In summary, we identified two $L M N A$ gene mutations in patients with EDMD2 and LGMD1B, which might be helpful to improve our current understanding of the genotype- phenotype correlation of the $L M N A$ gene mutations. To our knowledge, this is the first report of Korean EDMD2 and LGMD1B patients shown to have mutations of the $L M N A$ gene.

\section{References}

Bonne G, Di Barletta MR, Varnous S, Becane HM, Hammouda EH, Merlini L, Muntoni F, Greenberg CR, Gary F, Urtizberea JA, Duboc D, Fardeau M, Toniolo D, Schwartz K (1999) Mutations in the gene encoding lamin A/C cause autosomal dominant EmeryDreifuss muscular dystrophy. Nat Genet 21:285-288

Bonne G, Mercuri E, Muchir A, Urtizberea A, Becane HM, Recan D, Merlini L, Wehnert M, Boor R, Reuner U, Vorgerd M, Wicklein EM, Eymard B, Duboc D, Penisson-Besnier I, Cuisset JM, Ferrer X, Desguerre I, Lacombe D, Bushby K, Pollitt C, Toniolo D, Fardeau M, Schwartz K, Muntoni F (2000) Clinical and molecular genetic spectrum of autosomal dominant Emery-Dreifuss muscular dystrophy due to mutations of the lamin A/C gene. Ann Neurol 48:170180

Brown CA, Lanning RW, McKinney KQ, Salvino AR, Cherniske E, Crowe CA, Darras BT, Gominak S, Greenberg CR, Grosmann C, Heydemann P, Mendell JR, Pober BR, Sasaki T, Shapiro F, Simpson DA, Suchowersky O, Spence JE (2001) Novel and recurrent mutations in lamin $\mathrm{A} / \mathrm{C}$ in patients with Emery-Dreifuss muscular dystrophy. Am J Med Genet 102:359-367

Di Barletta MR, Ricci E, Galluzzi G, Tonali P, Mora M, Morandi L, Romorini A, Voit T, Orstavik KH, Merlini L, Trevisan C, Biancalana V, Housmanowa-Petrusewicz I, Bione S, Ricotti R, Schwartz K, Bonne G, Toniolo D (2000) Different mutations in the $L M N A$ gene cause autosomal dominant and autosomal recessive Emery-Dreifuss muscular dystrophy. Am J Hum Genet 66:14071412

Emery AEH (2000) Emery-Dreifuss muscular dystrophy - a 40-year retrospective. Neuromuscul Disord 10:228-232

Fatkin D, MacRae C, Sasaki T, Wolff MR, Porcu M, Frenneaux M, Atherton J, Vidaillet HJ, Spudich S, de Girolami U, Seidman JG, Seidman CE (1999) Missense mutations in the rod domain of the lamin A/C gene as causes of dilated cardiomyopathy and conduction-system disease. N Engl J Med 341:1715-1724

Genschel J, Schmidt HHJ (2000) Mutations in the LMNA gene encoding lamin A/C. Hum Mutat 16:451-459

Heald R, McKeon F (1990) Mutations of phosphorylation sites in lamin A that prevent nuclear lamina disassembly in mitosis. Cell 61:579-589

Kitaguchi T, Matsubara S, Sato M, Miyamoto K, Hirai S, Schwartz K, Bonne G (2001) A missense mutation in the exon 8 of lamin A/C gene in a Japanese case of autosomal dominant limb-girdle muscular dystrophy and cardiac conduction block. Neuromusc Disord 11:542546

Muchir A, Bonne G, van der Kooi AJ, van Meegen M, Baas F, Bolhuis PA, de Visser M, Schwartz K (2000) Identification of mutations in the gene encoding lamins $\mathrm{A} / \mathrm{C}$ in autosomal dominant limb girdle muscular dystrophy with atrioventricular conduction disturbances (LGMD1B). Hum Mol Genet 9:1453-1459

Nagano A, Arahata K (2000) Nuclear envelope proteins and associated diseases. Curr Opin Neurol 13:533-539

Shackleton S, Lloyd DJ, Jackson SNJ, Evans R, Niermeijer MF, Singh BM, Schmidt H, Brabant G, Kumar S, Durrington PN, Gregory S, O'Rahilly S, Trembath RC (2000) $L M N A$, encoding lamin A/C, is mutated in partial lipodystrophy. Nat Genet 24:153-156

Stuurman N, Heins S, Aebi U (1998) Nuclear lamins: their structure, assembly, and interactions. J Struct Biol 122:42-66

van der Kooi AJ, Ledderhof TM, de Voogt WG, Res JCJ, Bouwsma G, Troost D, Busch HFM, Becker AE, de Visser M (1996) A newly recognized autosomal dominant limb girdle muscular dystrophy with cardiac involvement. Ann Neurol 39:636-642 